\title{
The Application of Regression Analysis Method on the Linear Control of the Pavement on Concrete Beam Bridge with Wide Spans
}

\author{
Rongxiang Shan ${ }^{1}$, a \\ ${ }^{1}$ China Merchants Chongqing Communications Research \& Design Institute Co., \\ Ltd. ${ }^{1}$,Chongqing 400067 \\ a29051618@qq.com
}

\begin{abstract}
Key Words: Beam Bridge with wide spans, Pavement linear, Regression analysis method
Abstract: Cantilevered construction is adopted in most concrete bridges with wide spans. Because of such factors as shrinking and gradually deforming of concrete, constructive error, and constructive load, the pavement cannot be perfectly flat when the bridge is accomplished. So it is necessary to adjust the linear of pavement when the bridge is paved. The essay takes the Steady Bridge as an example, and adopts regression analysis method to work out the curve equation of pavement and to control the linear of bridge and the thickness of pavement. The practice proves a good turn-out, which provides a new thinking way to control the linear of pavement on the concrete beam bridge with wide spans.
\end{abstract}

\section{A General introduction}

At present, the method of coasting on cantilever by section is adopted in building concretemade continuous beam bridge with wide span and continuous rigid bridge. In process of building, the linear of the beam bridge is influenced by many certain and uncertain factors, such as physical and mechanical properties, shrinking and gradual deformation of concrete, precision of construction, construction load, temperature and so on so forth. Because there is always difference between the ideal the reality, those factors are to lead to a structural error in every phase from cantilever construction and closure and accumulation of those errors. To keep a good Longitudinal linear when the bridge is in service, remain the cross cut of pavement and enhance the safety and comfort of traffic, it is a must to adjust the elevation of the pavement according to the actual situation, fit the vertical linear of the pavement, and make sure that the bridge is paved evenly in the second period of the dead load pavement.

As for the bridge with narrow span, because most structures are precast or monolithic casting with a highly even top of the main beam and a smaller preset camber, the design requirements can be met by taking a uniformed thickness of pavement or method of simple fitting curve. Conventional fitting curve includes circular curve, vertical curve, three-point fitting parabola, five-point fitting parabola and other methods. Because of the unevenly paved bridge with a bigger preset arc in span, concrete-made bridge with coasting on cantilever by section in particular, it is possible to cause such problems as uneven thickness of pavement, a great difference in local parts in the bridge with wide span, which cannot satisfy the design and construction requirements. The essay takes the Steady Bridge as an example, and adopts regression analysis method to work out the curve equation of pavement and to control the linear of bridge and the thickness of pavement. The practice proves a good turn-out, which provides a new thinking way to control the linear of pavement on the concrete beam bridge with wide spans.

\section{Principle of vertical section linear fitting}

Basic principle

The most difficult contradiction when pavement is built in concrete-made bridge is among evenness, thickness and elevation, especially when the evenness of the main beam is poor. The chief cause is the designed thickness of the pavement ranges from $8 \mathrm{~cm}$ to $15 \mathrm{~cm}$. Over-thickness is 
not economic and adds deadweight, which is not good for the stress of the bridge structure. Thinness tends to cause cracks or damage in the early phase because of a lack of ability for load. for applicability, the altitude of the pavement should be adjusted and controlled based on the lower load layer of it. It is ideal that vertical section is a smooth curve with an even thickness compatible with the average and designed thickness after the concrete-made bridge is paved. Influenced by the objective factors, such an ideal situation is impossible to reach. In practice, "even, smooth and uniformed" is often to be considered and implemented, that is, on the premise that vertical section is kept smooth, pavement even and traffic smooth, we should control the thickness of pavement to agree with the designed, and try to make the thickness even.

\section{Principle of adjustment}

(1) When the paved thickness is designed to be $h \mathrm{~cm}$, the practical thickness should be controlled within $(h \pm 2) \mathrm{cm}$ and kept the whole volume of the paved layer unchanged in the period of pavement construction.

(2) Linear should be smooth. To evaluate the smooth of the linear, the concept of pavement evenness is brought in. Pavement evenness has a direct impact on the comfort of traffic, the duration of pavement service and every damage of vehicles, which exerts a great influence on the driving safety and economics on expressway.

(3) Keep the cross slope of pavement. It is better to remain the cross slope as it is built to avoid a sense of sloshing. but if the level on two sides is higher or lower because of every cause like construction, the cross section is needed to be adjusted within the requirements.

Location of the fitting curve

On basis of the adjusting principles above, with the influence caused by dead load in second phase, shrinking and gradual deformation and 1/2 live load considered, the dead load in the second phase and the variation produced by the shrinking and gradual deformation in concrete in later period are taken into consideration when the arc is preset. The maximum effect of the preset arc in cantilevered prestressed concrete bridge lies in the span, minimum in the support. When the realtime monitoring is fitted section by section, the intersection point of the fitting curve, as the controlling point of fitting curve equation, is taken in the middle of the pile or pile, because the level of the box beam near the middle part is influenced by the dead load in the second phase and he shrinking and gradual deformation in concrete in later period to the greatest degree, and the pile to the least. Besides, curve of middle span and side span should be fitted respectively.

\section{Regression analysis equation to solve the vertical linear equation of the bridge}

Basic principle

The preset arc in bridge is to guarantee the enough rigidity of bridge structure and to avoid an over deflection produced by dead weigh, shrinking and deformation of the concrete and other factors, which influence the traffic, leads to a damage to pavement and dangers the safety of the bridge. In the process of construction, preset reverse deflection makes the bridge inservice reach an ideal linear, so the preset arc of the section is an accumulation of every elements. Because the linear of the vertical section is only related with ordinate and abscissas, one-dimensional element regression is adopted. Since the preset arc in the vertical section is curve, it is hard to have a good fitting date by one-dimensional element regression model and the significance test can not be passed. As for a practical curve regression problem, it is common the depict the scattered points on rectangular coordinates according to monitored data to identify which kind of curve these points are similar to, and then conduct a calculation and significance test in line regression model. After this test is passed, curve regression equation is solved. 
A case of calculation

(1) The Steady Bridge is an extra-large bridge located in the second-phase of Expressway from Chongqing to Guizhou that lies in Anwen County, Lijiang Town in Chongqing, which crosses over Tiaoyu River. The type of the main bridge is a prestressed concrete continuous rigid bridge with three spans. the span is $122 \mathrm{~m}+210 \mathrm{~m}+122 \mathrm{~m}$. the main beam adopts variable thin-walled and wide box beam with single box and cell. The height of No. 0 beam on the top of pile and the foot of box beam is $13 \mathrm{~m}$. The height of the coating support in middle span and side span is $4.0 \mathrm{~m}$, changing as half-cube parabola. The width of the top of beam is $22.5 \mathrm{~m}$, and the bottom is $11.0 \mathrm{~m}$. C55 concrete is adopted and prestress in three directions is set in the beam. The designed load is that the car is super 20 degree and trailers 120 . The pavement is designed as $8 \mathrm{~cm}$ water-proof concrete evenadjusting layer, $5 \mathrm{~cm}$ mesograin asphalt concrete $+4 \mathrm{~cm}$ fine particle asphalt, to make sure asphalt concrete mechanic pavement is smooth and the keep the linear of the vertical section in later period. Fitting curve is employed to solve the level of cast-in-site water-proof reinforced concrete evenadjusting layer.

\section{(2) Fitting curve equation}

Because the number of preset arc is smaller compared to the horizontal coordinates, different parabola equation is adopted to combine and adjust in the process of fitting. Meanwhile, the times of fitting curve equation in middle span and side span should be the same or similar.

Take the curve equation in the middle as an example to introduce the steps of solving the fitting curve equation.

Firstly, we should measure the real elevation of the concrete on the top of main beam after the bridge is accomplished. With the cross slope considered, 3 monitor points should be set every half of bridge on every cross-section. A cross section is measured every each $10 \mathrm{~m}$. An average elevation of each monitor point is reached.

It is supposed that fitting curve equation is two-order parabola. Take the middle point of the span as the origin of coordinate. Three-point fitting parabola is employed to fit parabola equation in the top of the middle of the two piles to get the elevation of pavement corresponded with the monitoring point and real elevation. By comparison, analysis and results, the thickness of pavement near the middle of span and pile is smaller tan the theoretical one of $8 \mathrm{~cm}$, and the $1 / 4$ of span is bigger than that. Take the middle point of the pile as the origin of coordinate. Three-point fitting parabola is employed to fit parabola equation in middle point of span and mirror point to get the elevation of pavement corresponded with the monitoring point and real elevation. By comparison, analysis and results, the thickness of pavement near the middle of span and pile is smaller tan the theoretical one of $8 \mathrm{~cm}$, and the $1 / 4$ of span is bigger than that. Therefore it is supposed to take the middle point of the span as the origin of coordinate with three-point fitting parabola on the top of the two piles, the fitting curve equation is to be:

$$
y=\frac{1}{l^{2}}\left(a x^{2}+b \sqrt{ } x\right)
$$

$l^{\prime} \_$a half of the middle span

$y$ _ $y$ The under of the radial is positive.

To alter $y^{\prime}-\frac{y}{\sqrt{x}}, x^{\prime}=x^{\frac{3}{2}}$ by variation, the original equation is converted into line regression model:

To get the index of line regression equation above $a=0.345, b=0.655$, to replace formula(1) Curve regression equation (1) of even-adjusting layer paved by reinforced concrete is to be: 


$$
y=\frac{1}{l^{2}}\left(0.345 x^{2}+0.655 \sqrt{x}\right)
$$

(3) Calculating results

The calculated elevation of very cross section in even-adjusting layer paved by reinforced concrete is worked out from the curve equation above. In table.1, it can be seen that paved thickness by water-proof concrete calculated by fitting curve meets requirements. The average of the whole paved thickness by water-proof concrete is $8.04 \mathrm{~cm}$, and the quantity of the concrete is almost the same as the designed number. The whole weigh of the dead load in the second phase agrees with the designed number, which adjusts the linear of the pavement and lays foundation for that uniformed pavement by mechanics in asphalt and concrete and the ideal linear of pavement comes into being.

Table. 1 The calculated thickness of water-proof concrete pavement in each cross section of the middle of span

\begin{tabular}{|c|c|c|}
\hline mile pile No. & theoretical thickness $(\mathrm{cm})$ & addition \\
\hline 195 & 8.0 & $1 \#$ section on the top of the pile \\
\hline 205 & 6.3 & \\
\hline 215 & 7.1 & \\
\hline 225 & 8.4 & \\
\hline 235 & 8.6 & \\
\hline 245 & 6.5 & \\
\hline 255 & 7.4 & \\
\hline 265 & 8.2 & \\
\hline 275 & 7.0 & \\
\hline 285 & 9.7 & \\
\hline 295 & 9.0 & \\
\hline 300 & 8.0 & section of the middle of span \\
\hline 305 & 8.7 & \\
\hline 315 & 9.3 & \\
\hline 325 & 8.8 & \\
\hline 335 & 8.4 & \\
\hline 345 & 8.3 & \\
\hline 355 & 9.4 & \\
\hline 365 & 7.2 & \\
\hline 375 & 6.5 & \\
\hline 385 & 8.4 & \\
\hline 395 & 7.7 & \\
\hline 405 & 8.0 & 2\# section on the top of the pile \\
\hline
\end{tabular}




\section{Conclusion}

(1) The practice proves that because of the unevenly paved bridge with a bigger preset arc in span, concrete-made bridge with coasting on cantilever by section in particular, regression analysis method is adopted to work out the curve equation of pavement and to control the linear of bridge and the thickness of pavement. The practice proves a good turn-out, which provides a new thinking way to control the linear of pavement on the concrete beam bridge with wide spans.

(2) It is of great importance to select a suitable curve equation to conduct fitting when regression analysis is employed in the process of adjustment of the linear of vertical section for a completed bridge. Because the different curve equation fitting has a great impact on the precision of the results, to make a reasonable select of fitting curve equation is waited to further explore.

\section{Reference}

[1] Xu Junlan. A Surveillance on Construction of Bridge with Big Span [M]. Beijing: People's Jiaotong Press, 2000.

[2] Wu Changqi. Yang Jiahua. Han Fenghua. Construction of Prestressed Concrete Bridge [M]. Beijing: People's Jiaotong Prss, 1981.

[3] Sun Ronggui. Huang Wenying. Yi Xiangyun. Mathematical Statistics [M]. Chongqing: Press of Chongqing University ,1995.

[4] Fan Lichun. Bridge Construction (volume 1) [M]. Beijing: People's Jiaotong Prss, 2001. 